\title{
Diabetes Mellitus and Neuromuscular Blockade: Review
}

\author{
Armendariz-Buil Ignacio ${ }^{1^{*}}$, Lobato-Solores Felix ${ }^{1}$ and Aguilera-Celorrio Lucianob ${ }^{2,3}$ \\ ${ }^{1}$ Department of Anesthesiology, Resuscitation, and Pain Management, Hospital San Pedro, Logroño, Spain \\ ${ }^{2}$ Department of Anesthesiology and Critical Care, Hospital Universitario Basurto, Bilbao, Spain \\ ${ }^{3}$ Department of Radiology, Surgery and Physical Medicine, University of Basque Country, Spain
}

*Corresponding author: Armendariz-Buil Ignacio, Department of Anesthesiology, Resuscitation, and Pain Management, Hospital San Pedro, Logroño, Spain, Tel: 0034658643547; E-mail: nachoarm80@hotmail.com

Rec date: Feb 10, 2016; Acc date: March 18, 2016; Pub date: March 25, 2016

Copyright: ( 2016 Ignacio AB, et al. This is an open-access article distributed under the terms of the Creative Commons Attribution License, which permits unrestricted use, distribution, and reproduction in any medium, provided the original author and source are credited.

\begin{abstract}
Introduction and objectives: International Diabetes Federation (IDF) estimates that worldwide there are 415 million adults aged 20-79 with diabetes mellitus (DM). Although diabetes mellitus (DM) is a highly prevalent disease, only a handful of studies have examined the neuromuscular function in diabetic patients. Even more surprising is this data, if we think that neuromuscular blockade (NMB) is an essential part in the induction and maintenance of general anesthesia. NMB is induced by neuromuscular blocking agents (NMBA). The aim of this review is to update the knowledge of neuromuscular blockade in diabetes mellitus and its implications in clinical practice.
\end{abstract}

Material and Methods: Studies on neuromuscular function in DM were reviewed to establish the pathophysiological bases. Subsequently, all the literature that included clinical studies on neuromuscular block in diabetic patients was revised. Clinical key and PubMed data bases were used.

Results: The first pathophysiological studies date back to the 60s: Lawrence noted that nerve conduction in peripheral nerves was slower in diabetic patients, even when they had not yet developed clinical neuropathy. Different works found a significant prolongation in the parameters of recovery of neuromuscular function in diabetic patients after administration of vecuronium or rocuronium (amino-steroid NMBA). We also know that time to train-onfour (TOF) ratio 0.90 (DURTOF90) was delayed in patients with type $2 \mathrm{DM}$. This variable is related with the risk of residual neuromuscular block (RNMB). Monitoring and reversal of neuromuscular blockade are the main measures to prevent the occurrence of RNMB. Regarding the reversal of NMB, when neostigmine is used, the action time delayed appears in diabetic patients. However, time of reversal with sugammadex show no significant differences between type 2 diabetic patients and non-diabetic patients.

Conclusion: Diabetic patients, even though they have not developed clinical neuropathy, have delayed recovery time after administration of NMB. NMB monitoring and reversal are the main measures and do not prevent RNMB. Reversal with sugammadex (selective reversal of amino-steroid agents) seems not to show difference between diabetics and non-diabetics. Furthermore, there are still many unanswered questions in the field of research of NMB in diabetic population.

Keywords: Diabetes mellitus; Neuromuscular function; Neuromuscular blockade; Residual neuromuscular blockade; Neuromuscular reversal; Neuromuscular monitoring

\section{Introduction}

International Diabetes Federation [1] (IDF) estimates that there are now 415 million adults aged 20-79 with DM worldwide. By 2040 this will rise to 640 million. Although DM is highly prevalent in our environment and one of the most important challenges of the modern medicine, only a handful of studies have examined the neuromuscular function in diabetic patients. The shortage of publications in this area is still more surprising, if we consider that the NMB is one of the pillars in the administration of general anesthesia. NMB during surgery is used in tracheal intubation and to improve surgical conditions. In this review we analyse the evidence at our disposal to apply in daily clinical practice. NMB is induced by the administration of neuromuscular blocking agents (NMBA, also called neuromuscular blockers or relaxant agentes) causing paralysis of the affected skeletal muscles. This is accomplished either by acting presynaptically at nicotinic Ach receptors (nRAch) via the inhibition of ACh synthesis or release or by acting postsynaptically at the nRAch of the motor nerve end-plate. NMBA are classified in depolarizing and non-depolarizing agents by their different mechanism of action. Non-depolarizing agents antagonize the action of Ach in a competitive manner at the nRAch. They do not produce conformational changes in the receptor, unlike depolarizing drugs (e.g. succynilcholine). The non-depolarizing NMBA are divided into two groups by their different molecular structure: the benzylisoquinoline compounds (e.g. cisatracurium, atracurium, tubocurarine) and the aminosteroid compounds (e.g. vecuronium, rocuronium).

\section{Material and Methods}

A literature search was conducted in articles pertaining to neuromuscular function or neuromuscular blockade in diabetes mellitus. It included physiological and clinical studies in ClinicalKey (https://www.clinicalkey.es/) and PubMed (https:// 
www.ncbi.nilm.hin.gov/pubmed) with key words: diabetes mellitus and neuromuscular function, neuromuscular blockade, residual neuromuscular blockade, neuromuscular reversal and neuromuscular monitoring. We included randomized trials investigating pharmacodynamic effects of neuromuscular blockade agents in diabetes mellitus type 2 since 1940 s to present. Also we included relevant publications about pathophysiology and neuromuscular monitoring in diabetes mellitus. No language restriction. It is a narrative bibliographic review.

\section{Pathophysiological Changes of Diabetes Mellitus that can Interfere with Neuromuscular Blockade}

Five studies [2-6] show different changes in the neurophysiological parameters in diabetes mellitus. In diabetic nerve, conduction velocity of the action potential is decreased, the amplitude of action potentials, both sensory and motor, is smaller, and the latency time is elongated. Lawrence [7] found that conduction velocity measured by EMG in patients who had developed diabetic neuropathy was lower than in the diabetic patients without neuropathy. Moreover, in this same study we found that even in patients without neuropathy, nerve conduction of the ulnar, peroneal and radial nerve was altered. Another study found that the development of diabetic neuropathy is not parallel to renal failure and we can find changes in nerve conduction in the absence of renal failure [8]. Therefore, the absence of clinical neuropathy does not rule alterations in nerve conduction in diabetic patients.

If we focus on the neuromuscular junction (NMJ), the pathophysiological changes induced by DM appear more localized at presynaptic nerve ending than at the postsynaptic membrane. Constantini [9] observed alterations in glucose transport and release of Ach at the presynaptic nerve ending after 3.5 months of streptomycin induced DM in rats. The decrease in the number of quanta of Ach released in the synaptic cleft corresponded to a decrease of plate potential at the NMJ of diabetic rats. Schiller [10] studied the response in the NMJ of diabetic rats during high frequency stimulation. He concluded that the diabetic nerve has a resistance on the high frequency stimulation. Schiller associated this resistance with increasing $[\mathrm{K}+]$ extracellular. In 1990, Bowman [11] published the results of his research on presynaptic nRAch: nRAch these are responsible for an increased release of Ach in the synaptic cleft during high frequency stimulation of the presynaptic nerve ending. Therefore, it is conceivable that changes in the NMJ of diabetic rats during high frequency stimulation, that Schiller [10] observed, are due to a default presynaptic involvement in the release of Ach.

\section{Pharmacodynamic Parameters of Neuromuscular Blockade in Diabetes Mellitus}

All the clinical studies published until this moment included diabetic patients without neuropathy or nephropathy diagnosed. In 1992, Atallah [12] was the first researcher who studied the effect of NMBA in patients with DM. He administered repeated doses of 0.25 $\mathrm{mg} / \mathrm{kg}$ of tubocurarine. Atallah did not monitor the usual parameters of NMB and tubocurarine is a disused relaxant. But this work is valuable because it marked the first clinical study in diabetic patients. Later, Saitoh [13,14], Alper [15] and Topal [16] found an extension in the duration of action (Dur90\%), clinical duration (Dur25\%) and recurrence times T1, T2, T3 and T4 of the train of four (TOF) in DM patients Type 2 compared to non-diabetics after administration of rocuronium and vecuronium. However, no differences were observed in onset time. In fact, in all the work reviewed, the differences in the times of recovery from neuromuscular block among diabetics and non-diabetics become more evident in the late stages of neuromuscular block reversal. This phenomenon can be explained by the decrease of Ach released in the synaptic cleft. If there is a decrease of Ach, spontaneous reversal will be delayed because there is less Ach to compete with the NMBA. From there, the differences found in the DURTOF70 and DURTOF90 (blocking recovery time to a TOF ratio $\geq$ 90 after the administration of a bolus dose of NMBA being significantly prolonged in type 2 diabetic patients compared with nondiabetics. These differences in the DURTOF90 have been observed both rocuronium [17] and vecuronium [18]. This parameter is strongly related to the risk of RNMB.

\section{Risk of Residual Neuromuscular Blockade in Diabetics}

Postoperative residual neuromuscular blockade in conscious patients in the recovery room is a well-recognized phenomenon that may increase postoperative morbidity [19]. No studies comparing the incidence of RNMB in diabetic patients to the general population are published. However, we do know that this population has more risk of RNMB. Two papers $[17,18]$ have reported a prolonged time to TOF ratio 0,9 (DURTOF90: variable related to the risk of RNMB) in patients with type $2 \mathrm{DM}$, one another using rocuronium (published by our Research Group) and vecuronium. Both studies included only diabetic patients without clinical neuropathy, renal failure or other diseases that could interfere with the NMB. Furthermore, within these diabetic patients a worse glycemic control was not observed expressed by glycated hemoglobin, this involves a greater risk of RNMB [17]. Therefore, in type 2 diabetic patients without neuropathy, we cannot know which patients are more predisposed to RNMB.

\section{RNMB Prevention in Diabetic Patients}

As for any patient, correct monitoring of neuromuscular blockade and adequate reversal are the two pillars to prevent RNMB [20].

\section{NMB Monitoring in Diabetic Patients}

Monitoring of NMB should be part of standard monitoring in any surgery which neuromuscular blocking agents is used. In the presence of DM, NMB monitoring is even more justified, if possible, by altering the parameters of neuromuscular function and increased risk of RNMB. It has been shown that quantitative monitoring can reduce the incidence of RNMB [20,21]. For optimum monitoring, it is advisable to calibrate the device (neurostimulator) prior to administration of NMBA and maintain the stimulation throughout the surgery. In diabetic patients, the supramaximal stimulus is of greater intensity than in the non-diabetic, even if not developed clinical neuropathy $[13,14]$. This can hinder the automatic calibration with standard devices [22]. If there is diabetic neuropathy, this problem is exacerbated succeeding calibration in less than $60 \%$ of patients [23]. In these cases, when calibration is not possible the device monitoring the adductor pollicis muscle after ulnar nerve stimulation, can be a good alternative monitoring orbicularis oculi muscle by stimulation of the facial nerve; cranial nerves are less affected in distal polyneuropathy, the most common form of presentation of diabetic neuropathy [24].

\section{Reversal of Neuromuscular Blockade in Diabetic Patients}

The reversal of NMB in the diabetic population has been little studied. Anticholinesterase drugs were the only reversers at our disposal until very recently. Of these, neostigmine is the most used and 
Page 3 of 4

studied. Saitoh [25] in 2004, noted that the number of diabetic patients with TOF ratio $<0.9$ was significantly greater than non-diabetics 15 minutes after neostigmine administration. Considering the pathophysiological changes described above, if less Ach is released, the reversion provided with anticholinesterase drugs will be slower because it is a competition between the concentration of NMB and Ach. The inevitable conclusion is that the reversal with neostigmine in diabetic patients is less effective.

But, what happens if we use sugammadex for the reversal in type 2 $\mathrm{DM}$ ? Sugammadex is a recent selective neuromuscular reversal agent. Its mechanism of action is different from anticholinesterase drugs. It acts by encapsulating amino steroids: such as rocuronium or vecuronium resulting faster than neostigmina reversal [26]. Furthermore, sugammadex does not have side effects of anticholinesterase drugs (e.g., Bradycardia, bronchospasm, respiratory secretions Increased, emesis). Our Research Group, in a study recently published [27], compared the effect of sugammadex in a group of diabetic patients versus a control group. The sugammadex was administered at the time of the reappearance of the second TOF response (T2) and measured the time until a TOF ratio $\geq 0.9$. We found no differences in time reversal with sugammadex in patients with type 2 diabetes compared to non-diabetics. These results make sense if we analyze the mechanism of action of sugammadex: it encapsulates rocuronium molecule to eliminate it, while neostigmine increases ACh in the synaptic cleft to compete with the neuromuscular blocker. While waiting for new data confirming these results, we can think that the reversal with sugammadex is safe in diabetic population who has not developed neuropathy.

\section{Halogenated in the Management of NMB in Diabetic Patients}

Saitoh [14] found that the TOF ratio 120 minutes after a dose of vecuronium was lower in diabetic patients anesthetized with sevoflurane than in diabetics anesthetized with propofol-TIVA (Total Intravenous Anesthesia). This finding leads us to think that the effects of DM and halogenated agents at the NMJ are additive. Furthermore, as it occurs with diabetes mellitus, the halogenated neuromuscular blocking effect becomes more apparent in long duration surgeries [28]. Therefore, in these interventions we must closely monitor NMB.

\section{Where You can Get Research?}

There is a large field of research in the area of NMB in diabetics. Future studies should address the neuromuscular function in type 1 diabetic patients, those with clinical neuropathy or diabetic nephropathy diagnosed. Furthermore, the reversal of neuromuscular block in diabetic patients can be studied more deeply. Moreover, the real incidence of RNMB has not been determined in the diabetic population.

\section{Conclusions}

Diabetic patients, even though they have not developed clinical neuropathy, have delayed recovery of neuromuscular function after administration of NMB. In addition, parameters related with an increased risk of RNMB are prolonged in diabetic patients. Monitoring neuromuscular blockade has been shown to prevent the appearance of $\mathrm{RNMB}$ in general population. Therefore, monitoring of $\mathrm{NMB}$ in diabetics is even more justified if possible, by altering pharmacodynamics parameters related to the risk of RNMB. Reversal with sugammadex seems not to show differences between diabetics and non-diabetics. On the contrary, neostigmine has a delayed time of action in patients with DM. Furthermore, there are still many unanswered questions in the field of research of neuromuscular blockade in the diabetic population.

\section{References}

1. International Diabetes Federation (2015) Diabetes atlas (7thedn). International Diabetes Federation.

2. Brown MJ, Sumner AJ, Greene DA, Diamond SM, Asbury AK (1980) Distal neuropathy in experimental diabetes mellitus. Ann Neurol 8: 168-178.

3. Sima AA, Hay K (1981) Functional aspects and pathogenetic considerations of the neuropathy in the spontaneously diabetic BB-Wistar rat. Neuropathol Appl Neurobiol 7: 341-350.

4. Mulder DW, Lambert EH, Bastron JA, Sprague RG (961) The neuropathies associated with diabetes mellitus. A clinical and electromyographic study of 103 unselected diabetic patients. Neurology 11: 275-284.

5. Tackmann W, Kaeser HE, Berger W, Rüeger AN (1981) Sensory and motor parameters in leg nerves of diabetics: intercorrelations and relationships to clinical symptoms. Eur Neurol 20: 344-350.

6. Tackmann W, Kaeser HE, Berger W, Rüeger AN, Violier E (1981) Autonomic disturbances in relation to sensorimotor peripheral neuropathy in diabetes mellitus. J Neurol 224: 273-281.

7. Lawrence DG, Locke S (1961) Motor nerve conduction velocity in diabetes. Arch Neurol 5: 483-489.

8. Lamontagne A, Buchthal F (1970) Electrophysiological studies in diabetic neuropathy. J Neurol Neurosurg Psychiatry 33: 442-452.

9. Constantini S, Schiller Y, Cohen AM, Rahamimoff R (1987) Pathophysiology of the neuromuscular junction in diabetic rats. Isr J Med Sci 23: 101-106.

10. Schiller Y, Rahamimoff R (1989) Neuromuscular transmission in diabetes: response to high-frequency activation. J Neurosci 9: 3709-3719.

11. Bowman WC, Prior C, Marshall IG (1990) Presynaptic receptors in the neuromuscular junction. Ann N Y Acad Sci 604: 69-81.

12. Atallah MM, Daif AA, Saied MM, Sonbul ZM (1992) Neuromuscular blocking activity of tubocurarine in patients with diabetes mellitus. Br J Anaesth 68: 567-569.

13. Saitoh Y, Kaneda K, Hattori H, Nakajima H, Murakawa M (2003) Monitoring of neuromuscular block after administration of vecuronium in patients with diabetes mellitus. Br J Anaesth 90: 480-486.

14. Saitoh Y, Hattori H, Sanbe N, Nakajima H, Akatu M, et al. (2005) Delayed recovery of vecuronium neuromuscular block in diabetic patients during sevoflurane anesthesia. Can J Anaesth 52: 467-473.

15. Alper I, Ulukaya S, Makay O, Balcioglu T (2010) The pharmacodynamic effects of rocuronium during general anesthesia in patients with type 2 diabetes mellitus. Minerva Anestesiol 76: 115-119.

16. Topal A, Sargin M, Tayfun Et, Uzun ST (2014) Neuromuscular Characteristics of Rocuronium in Diabetic Patients. Cukurova Med J 39: 807-813.

17. Armendáriz-Buil I, Lobato-Solores F, Aguilera-Celorrio L, Morros-Díaz E, Fraile-Jiménez E, et al. (2014) Residual neuromuscular block in type II diabetes mellitus after rocuronium: a prospective observational study. Eur J Anaesthesiol 31: 411-416.

18. Nitahara K, Sugi Y, Shigematsu K, Haraga I, Abe S, et al. (2013) Recovery of train-of-four ratio to 0.70 and 0.90 is delayed in type 2 diabetes with vecuronium-induced neuromuscular block. Eur J Anaesthesiol 30: 80-84.

19. Berg H, Roed J, Viby-Mogensen J, Mortensen CR, Engbaek J, et al. (1997) Residual neuromuscular block is a risk factor for postoperative pulmonary complications. A prospective, randomised, and blinded study of postoperative pulmonary complications after atracurium, vecuronium and pancuronium. Acta Anaesthesiol Scand 41: 1095-1103. 
Citation: Ignacio BA, Solores FL, Lucianob CA (2016) Diabetes Mellitus and Neuromuscular Blockade: Review. J Diabetes Metab 7: 678. doi: 10.4172/2155-6156.1000678

Page 4 of 4

20. Baillard C, Clec'h C, Catineau J, Salhi F, Gehan G, et al. (2005) Postoperative residual neuromuscular block: a survey of management. $\mathrm{Br}$ J Anaesth 95: 622-626.

21. Murphy GS, Szokol JW, Marymont JH, Greenberg SB, Avram MJ, et al. (2008) Intraoperative acceleromyographic monitoring reduces the risk of residual neuromuscular blockade and adverse respiratory events in the postanesthesia care unit. Anesthesiology 109: 389-398.

22. Iwasaki H, Takahoko K, Otomo S, Sasakawa T, Kunisawa T, et al. (2013) Evaluation of neuromuscular blockade using TOF-Watch SX in type 2 diabetes patients. Masui 62: 929-934.

23. Knüttgen D, Burgwinkel W, Nieden KZ, Jahn M, Müller-Gorges MR, et al. (1996) Limited applicability of the DATEX Reloxograph in diabetics with peripheral polyneuropathy. Int J Clin Monit Comput 13: 21-25.

24. Unterbuchner C, Fink H, Berthele A, Blobner M (2014) Case scenario: residual curarization in diabetic polyneuropathy. Anesthesiology 120: 474-479.
25. Saitoh Y, Hattori H, Sanbe N, Nakajima H, Akatu M, et al. (2004) Reversal of vecuronium with neostigmine in patients with diabetes mellitus. Anaesthesia 59: 750-754.

26. Paton F, Paulden M, Chambers D, Heirs M, Duffy S, et al. (2010) Sugammadex compared with neostigmine/glycopyrrolate for routine reversal of neuromuscular block: a systematic review and economic evaluation. Br J Anaesth 105: 558-567.

27. Armendariz-Buil I, Lobato-Solars F, Aguilera-Celorrio L, Zabala DD, Londoño AG, et al. (2015) Neuromuscular block reversal with sugammadex in type 2 diabetic patients. Perioperative Care and Operating Room Management 1: 13-18.

28. Lowry DW, Mirakhur RK, McCarthy GJ, Carroll MT, McCourt KC (1998) Neuromuscular effects of rocuronium during sevoflurane, isoflurane, and intravenous anaesthesia. Anesth Analg 87: 936-940. 